\title{
Time-Resolved Reflectance Spectroscopy Applied to the Nondestructive Monitoring of the Internal Optical Properties in Apples
}

\author{
RINALDO CUBEDDU, COSIMO D'ANDREA, ANTONIO PIFFERI, \\ PAOLA TARONI, ALESSANDRO TORRICELLI, GIANLUCA VALENTINI, \\ MARGARITA RUIZ-ALTISENT, CONSTANTINO VALERO, CORAL ORTIZ, \\ COLIN DOVER, and DAVID JOHNSON \\ INFM-Dipartimento di Fisica, and CEQSE-CNR, Politecnico di Milano, Piazza Leonardo da Vinci 32, 20133 Milan, Italy (R.C., \\ C.D., A.P., P.T., A.T., G.V.); Rural Engineering Department, E.T.S.I. Agronomos, Polytechnic University of Madrid, Av. \\ Complutense, 28040 Madrid. Spain (M.R.A., C.V., C.O.); and Horticulture Research International (HRI), East Malling. West \\ Malling, Kent, ME19 6BJ, UK (C.D., D.J.)
}

\begin{abstract}
Time-resolved reflectance has been used for the nondestructive measurement of optical properties in apples. The technique is based on the detection of the temporal dispersion of a short laser pulse injected into the probed medium. The time distribution of re-emitted photons interpreted with a solution of the diffusion equation yields the mean values of the absorption and reduced scattering coefficients of the medium. The proposed technique proved useful for the measurement of the absorption and scattering spectra of different varieties of apples, revealing the spectral shape of chlorophyll. No major variations were observed in the experimental data when the fruit was peeled, showing that the optical properties measured were those of the pulp. With this technique the change in chlorophyll absorption during storage and ripening could be followed. Finally, a compact prototype working at few selected wavelengths was designed and constructed, demonstrating potentialities of the technique for industrial applications.
\end{abstract}

Index Headings: Chlorophyll; Absorption; Scattering; Photon migration; Fruit.

\section{INTRODUCTION}

The assessment of the internal quality of fruits is of increasing importance in an ever more competitive market. Further, there is an increasing demand for nondestructive and noninvasive tests to determine the optimum time for harvesting, to follow changes during storage, and to determine the internal quality of individual fruit.

Different nondestructive techniques have been proposed to probe a variety of quality-related factors in fruits. ${ }^{1}$ For example, anthocyanins in strawberries have been detected by photoacoustic techniques. ${ }^{2}$ The artificial nose, with the potential to detect small quantities of released chemicals, may prove useful for those aspects of quality related to aroma production. ${ }^{3}$ even though few data on such applications are currently available. Ultrasounds cannot penetrate deeply into the pulp of most fruits due to the porous nature of the tissue, yet some promising results were obtained by using low-frequency ultrasounds. ${ }^{4}$ Nuclear magnetic resonance appears promising in terms of specificity and spatial resolution, ${ }^{5}$ but is not suitable for in-the-field or mass applications.

Other techniques using visible or near-infrared light have been devised on the basis of the measurement of the total diffusely reflected light at different wavelengths. For instance, in the visible region of the spectrum, colorimetry has been used to determine the color of the skin of peaches ${ }^{6}$ and, in the near-infrared region, the spectrum of re-emitted light has been studied mainly to estimate the total sugar content.?

A key limitation of these optical techniques is that the intensity of the diffusely re-emitted light is strongly dependent on the color of the skin, thus masking information from the pulp. Moreover, the total reflected intensity is determined by both the absorption and the scattering properties, in such a way that it is not feasible to separate the effects of these properties. Absorption and scattering contain distinct information on the medium. Absorption is determined by the pigments and constituents of the pulp that produce characteristic spectral features in the visible and near-infrared region of the spectrum. Conversely, scattering is due to the local variation of the dielectric constant inside the medium. Microscopic changes in refractive index caused by membranes, air vacuoles. or organelles deviate the photon paths and are ultimately responsible for light diffusion. Macroscopically, this phenomenon can be described by using the scattering coefficient $\left(\mu_{\mathrm{s}}\right.$ ) that corresponds to the mean distance between interaction sites and the angular probability distribution of scattered photons. A further simplification assumes a single parameter, the transport or reduced scattering coefficient $\mu_{s}^{\prime}=\mu_{s} \cdot(1-g)$, where $g$ is the mean cosine of the scattering angle for a single scattering event. This parameter corresponds to the effective scattering coefficient, assuming isotropic scattering, and is adequate to describe light distribution for highly scattering media (e.g., apples). ${ }^{8}$

An interesting challenge for the noninvasive simultaneous measurement of the scattering and the absorption properties of turbid media is offered by time-resolved reflectance spectroscopy (TRS). This technique is based on the measurement of the temporal delay and broadening experienced by a short laser pulse while traveling through a turbid medium. ${ }^{9}$ Usually, the laser light is injected into and collected from the medium by using two optical fibers placed in contact with the surface at a fixed relative distance. If an appropriate theoretical model is used for the analysis of the experimental data, it is possible to accurately measure both the absorption coefficient $\left(\mu_{\mathrm{a}}\right)$ 


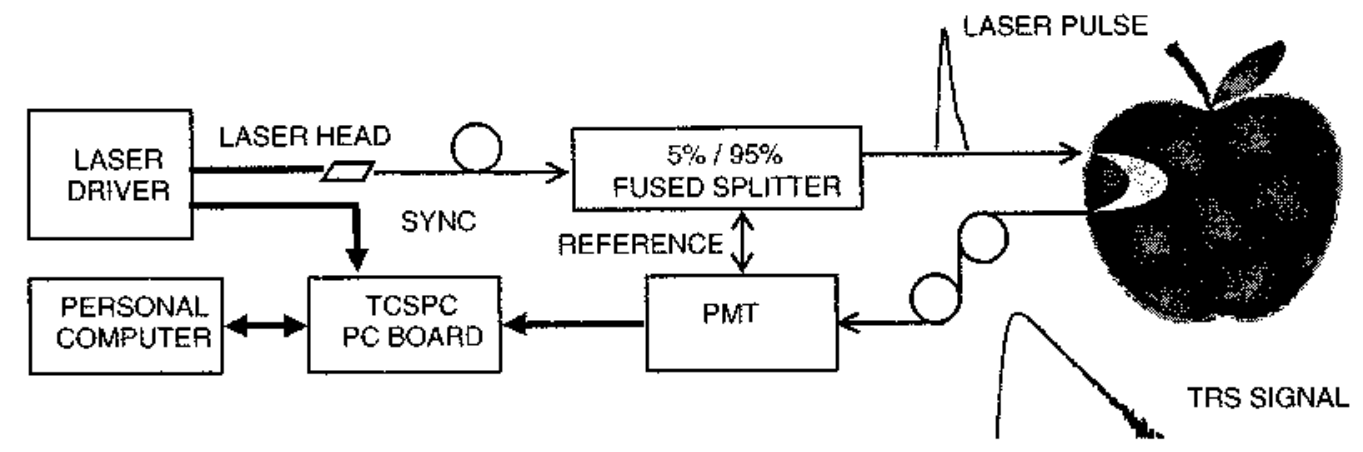

FIG. 1. Layout of the compact prototype based on a semiconductor pulsed laser, fiber optics for signal injection and collection, a metal channel compact size photomultiplier (PMT), and a computer board for time-correlated single photon counting (TCSPC).

and the reduced scattering coefficient of the probed medium. 10,11

This technique has been successfully used for the detection of optical properties in biological media for biomedical applications. ${ }^{12}$ Recently, we have demonstrated ${ }^{13}$ its applicability for the nondestructive measurement of absorption and scattering spectra in different fruits and vegetables (apple, kiwifruit, peach, tomato). In this paper we explore the key features of this novel approach. in particular in view of possible applications on agriculture produce, using the apple as a test model. Relevant issues such as influence of the skin properties or extension of the region involved in the measurement will be analyzed. together with tests on sensitivity of the technique to physiological changes in the fruit. Finally, a first implementation of the technique with a compact instrument will be presented.

\section{MATERIALS AND METHODS}

Instrumentation: Laboratory Setup. Spectral measurements were obtained with the use of a laboratory system for time-resolved reflectance spectroscopy. A synchronously pumped mode-locked dye laser was used as the illuminating source. The dye (DCM) was pumped by an actively mode-locked argon laser (CR-18. Coherent, CA). The repetition rate of the pulses was reduced to about $9 \mathrm{MHz}$ by means of a cavity dumper. The dye laser is tunable between 610 and $700 \mathrm{~nm}$, with an average power of about $10 \mathrm{~mW}$ and a pulse width of $\leq 20$ ps. The laser light was injected into and collected from the sample by means of $1-\mathrm{mm}$-core $1-\mathrm{m}$-long plastic-glass fibers set on the fruit surface at a relative distance of 1.5 cm. A fiber holder kept the fibers in parallel and in contact with the sample and prevented direct collection of specular reflected light. The distal end of the collecting fiber was placed at the entrance slit of a scanning monochromator (HR-250. Jobin Yvon. France), coupled to a double microchannel plate photomultiplier (R1564U, Hamamatsu. Japan). The signal was processed by an electronic chain for time-correlated single-photon counting. The triggering signal was provided by coupling part of the laser light to a fast photodiode. A small fraction of the incident beam was coupled to another fiber and directly fed to the entrance slit of the monochromator to compensate for any temporal drift of the electronic chain. The temporal width of the instrumental response function (IRF) was $<120$ ps (full width at half-maximum), mea- sured by direct contact between the injection and collection fibers. The system was controlled by a PC that facilitated the automatic acquisition of a set of time-resolved reflectance curves over a given wavelength range. Typically, the overall tine required to acquire a set of time-resolved reflectance measurements from 610 to 700 nm every $5 \mathrm{~nm}$ with 100000 counts per curve was about $1 \mathrm{~min}$.

Instrumentation: Compact Prototype. A compact prototype for TRS measurement suitable for application on fruits was developed at Politecnico di Milano. The scheme of the instrument is shown in Fig. 1. Light is provided by a pulsed laser diode (PDL 800. Picoquant. Germany) running at $80 \mathrm{MHz}$ with an average power of $1 \mathrm{~mW}$ and a typical pulse width of $100 \mathrm{ps}$. Different laser heads can be interchanged, permitting selection of the illuminating wavelength. Results presented in this study were obtained by using a diode emitting at $672 \mathrm{~nm}$. Light was coupled into a fused fiber splitter (FUSEDIRVIS $5 / 95, \mathrm{OZ}$ Optics, Canada) that conveyed most of the power $(95 \%)$ to the fruit and a small fraction $(5 \%)$ to the detector for continuous monitoring of the IRF. Re-emitted photons were collected at a distance of $2 \mathrm{~cm}$ from the injection point by a $1 \mathrm{~mm}$-core plastic fiber and redirected to a compact metal channel diode photomultiplier (R5600U-L16. Hamamatsu, Japan). The signal was then processed by an integrated PC board for time-correlated single-photon counting (TimeHarp, Picoquant, Gernany). The typical acquisition time was $4 \mathrm{~s}$ per measurement point. All the equipment can be easily hosted in a small box and operated by a standard PC.

Analysis. The temporal profile of the TRS curve provided by either of the two instruments was analyzed by using a solution of the radiative transport equation under the diffusion approximation for a semi-infinite homogeneous medium. ${ }^{10}$ The extrapolated boundary condition was used ${ }^{14}$ to take into account the refractive index mismatch at the surface. The reflectance $R(r, t)$-i.e., the photon probability of being re-enitted from the tissue at a time $t$ and a distance $r$ from the injection-can be expressed as in Eq. 1:

$$
\begin{aligned}
R(r, t)= & A D^{-3 / 2} t^{-5 / 2} \exp \left(-\mu_{\mathrm{a}} v t\right) \exp \left(-\frac{r^{2}}{4 D v t}\right) \\
& \times\left[z_{0} \exp \left(-\frac{z_{0}^{2}}{4 D v t}\right)-z_{\mathrm{p}} \exp \left(-\frac{z_{\mathrm{p}}^{2}}{4 D v t}\right)\right]
\end{aligned}
$$




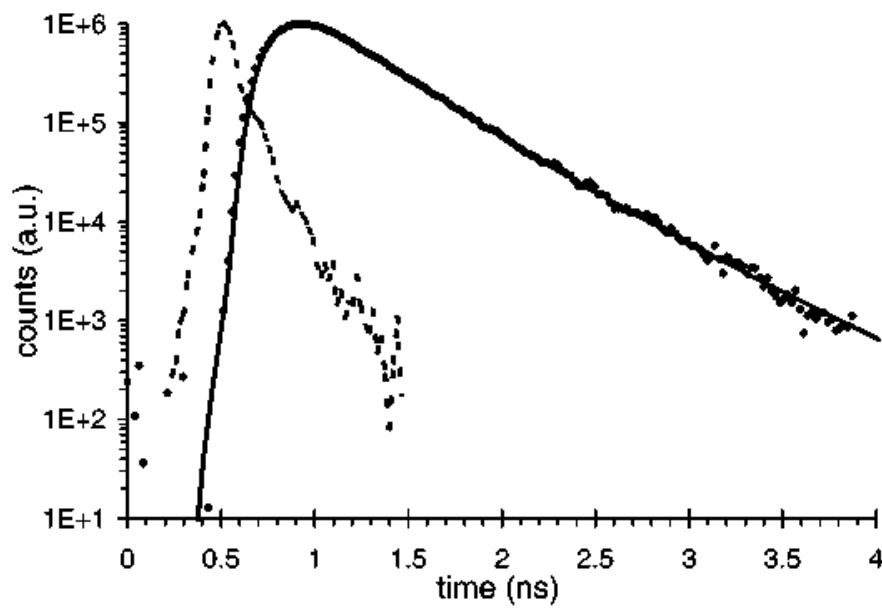

Fig. 2. Typical fit of a time-resolved reflectance curve. Experimental data (dots) are fitted with the convolution (continuous line) of the instrumental transfer function (dashed line) with a theoretical model (not shown).

where $A$ is a normalization constant; $v=c / n$ is the speed of light in the medium; and $z_{0}=1 / \mu_{s}^{\prime}$ is the scattering mean free path: while $z_{\mathrm{p}}$ derives from the extrapolated boundary conditions and depends on the refractive index of the tissue. The diffusion coefficient $D=1 /\left(3 \mu_{s}^{\prime}\right)$ was taken to be independent of the absorption properties of the medium. ${ }^{15}$

The experimental curve was fitted with a convolution of the theoretical function with the IRF. The best fit was reached by minimizing the $\chi^{2}$, varying both $\mu_{\alpha^{*}}$ and $\mu_{s}^{\prime}$ using a Levenberg Marquard iterative procedure. ${ }^{16}$ The range of the fit included all the points of the experimental curve with a number of counts higher than $10 \%$ of the peak value on the rising edge of the curve and $1 \%$ of the peak value on the falling edge. Figure 2 shows the best fit of a typical experimental curve. The IRF is also shown for comparison (dashed line). The scattering properties of the medium yield the temporal shift and broadening of the reflectance curve as compared to the transfer function, while the absorption properties determine the slope of the tail in a logarithmic plot. The fitting procedure can automatically analyze a full batch of experimental curves on a standard PC at a speed of 10 curves per second. Synchronization of the analysis PC with the measurement one over a network permits on-line processing of the experimental data so that the absorption and scattering spectra are shown on the screen in real time while the measurement is in progress.

The accuracy of a TRS measurement for the detection of $\mu_{\mathrm{s}}$ and $\mu_{\mathrm{s}}^{\prime}$ in a turbid medium has been already discussed elsewhere. ${ }^{17,18}$ In general, for a given set of $\mu_{\mathrm{a}}$ and $\mu_{s}^{\prime}$ values, the accuracy of the measurement increases with the interfiber distance $\rho$, provided that enough photons can be collected (about 100000). We have shown that, for $\rho=1.4 \mathrm{~cm}, \mu_{s}^{\prime}=10 \mathrm{~cm}^{-1}$, and $\mu_{\mathrm{a}}<0.25 \mathrm{~cm}^{-1}$. the measurement of $\mu_{\mathrm{a}}$ is accurate within $10 \%$, while the measurement of $\mu_{s}^{\prime}$ is accurate within $20 \% .^{18}$ For $\rho=1.5$ $\mathrm{cm}$ as used in the present study, and a $\mu_{s}^{t}$ value around $10 \mathrm{~cm}^{-1}$ or higher, a comparable or better accuracy can be expected.

Fruit Samples. Apples of the Golden Delicious, Granny Smith, and Starking Delicious varieties, used for the characterization of the technique, were taken from the local market. Fruits involved in the picking date experiment performed with the compact prototype were taken from controlled orchards at Horticulture Research International (UK). A total of 30 apples of the Gala variety were harvested on three different occasions during September/October and then stored in refrigerated controlled atmospheric conditions until early May when all fruits were characterized within the same measurement session.

\section{RESULTS}

The absorption and scattering spectra obtained from intact apples of three different varieties (Golden Delicious, Granny Smith, and Starking Delicious) are shown in Fig. 3. For each variety, two fruits of different maturity (green and yellow background color, respectively) were measured and, for each fruit, two sides were probed: the side most exposed to sunlight and the opposite side. This was done to highlight the variation in optical properties observed between and within fruits. The main feature of the absorption spectra is the peak around $675 \mathrm{~nm}$. which corresponds to absorption by chlorophyll-a (CHL). Considerable variation between different varieties is evident, with the less mature fruit showing more CHL absorption, particularly for Granny Smith. In general, there was less CHL absorption for the side of the fruit most exposed to sunlight; this difference was greater for the less mature fruit of each variety, and the inter-fruit variation was lower than the intra-fruit variability. In apples with quite a low CHL absorption (e.g., Golden, Triangles), the absorption spectrum was characterized by a slightly decreasing line shape that is probably the tail of an anthocyanine spectral peak. Water absorption is negligible in this wavelength range $\left(<0.005 \mathrm{~cm}^{-1}\right.$ for pure water).

Scattering spectra of the same fruits are presented in the right pane. No particular spectral features were evident. However, a slight decrease in $\mu_{s}^{\prime}$ with increasing wavelength was observed. Starking Delicious apples had higher $\mu_{s}^{\prime}\left(13-22 \mathrm{~cm}^{-1}\right)$ than Golden Delicious or Granny Smith apples $\left(12-15 \mathrm{~cm}^{-1}\right.$ and $8-13 \mathrm{~cm}^{-1}$, respectively). Variation in scattering coefficient was found between different fruits of the same variety and different positions on the same apple. There seened not to be any relationship between CHL absorption and the scattering spectra. Yet, the number of individual fruits analyzed is too small to draw any conclusion.

The influence of the skin on the result of a TRS measurement is shown in Fig. 4. Absorption and scattering spectra were obtained for a Golden Delicious, a Granny Smith, and a Starking Delicious apple. Each fruit was measured before and after skin removal with the fibers positioned at the same point to probe the same region. The spectra obtained for the whole apples overlap the spectra for the peeled apples for both absorption and scattering. Thus, it is possible to infer that the TRS measurement probes the optical properties of the pulp of the fruit regardless of the skin color.

In a further experiment, the penetration depth of a TRS measurement was determined. It is well known that the volume probed by a TRS measurement is a "banana shaped" region connecting the injection and collection points. ${ }^{19}$ It is difficult to define the measurement volume. 

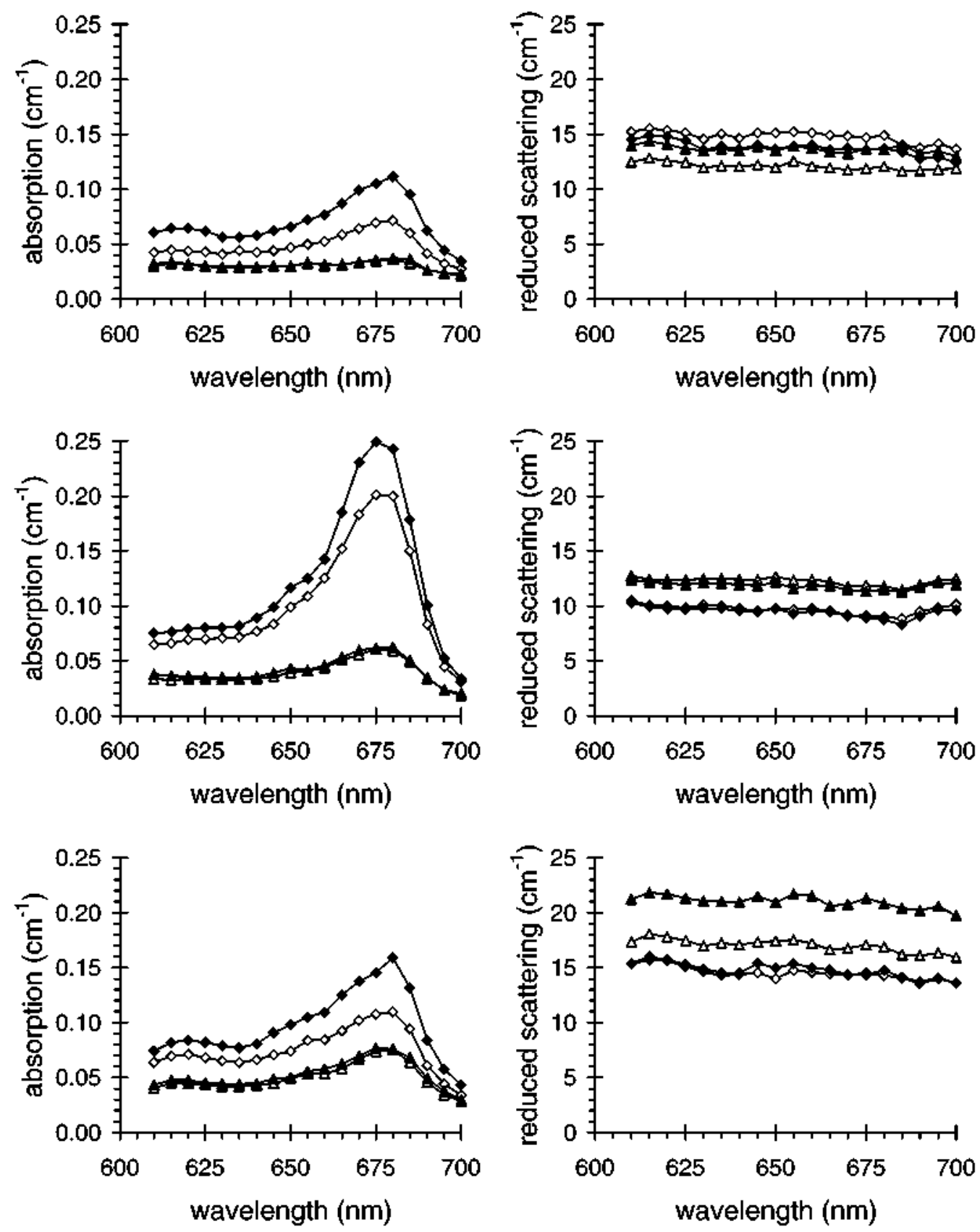

FIG. 3. Absorption (left pane) and scattering (right pane) spectra of Golden Delicious (top row), Granny Smith (middle row), and Starking Delicious apples (bottom row). Yellow background color (triangles). green background color (diamonds). Side more exposed to sunlight (empty symbols), opposite side (filled symbols).

as photon paths can be distributed in the whole medium. although they are more densely packed in the banana region. Thus, an attempt was made to determine the maxinum depth in the pulp for which there was some detectable contribution to the TRS curve. A series of measurements were performed on a Starking Delicious apple. with slices of pulp cut from the side of the fruit opposite to that in contact with the fibers. Spectra were taken from the whole apple and then from tissue with a total thickness of $4.1 .2 .7,2.1$, and $1.5 \mathrm{~cm}$. The fitted absorption and scattering spectra are shown in Fig. 5. The absorption coefficient was unchanged down to a thickness of $2.7 \mathrm{~cm}$. At $2.1 \mathrm{~cm}, \mu_{\mathrm{a}}$ had increased by $25 \%$ at $675 \mathrm{~mm}$ from the measurement on the whole apple, while at a thickness of
$1.5 \mathrm{~cm}$ the increase was $50 \%$. The greatest differences were observed on the tails of the spectrum, where absorption was lower. The results for the scattering coefficient show similar behavior, with almost no change down to a thickness of $2.7 \mathrm{~cm}$, and increases of $15 \%$ and $25 \%$ for 2.1 and $1.5 \mathrm{~cm}$ thickness, respectively.

To determine the sensitivity of the technique, we followed the change in chlorophyll absorption as a result of storage at room temperature. Two Golden Delicious apples at different ripeness stages were measured at different times (time 0 , and after 4 days of storage at room temperature). The results are reported in Fig. 6. In both apples there is a clear decrease in the absorption coefficient, related to a decrease in CHL content, of about $30 \%$ 

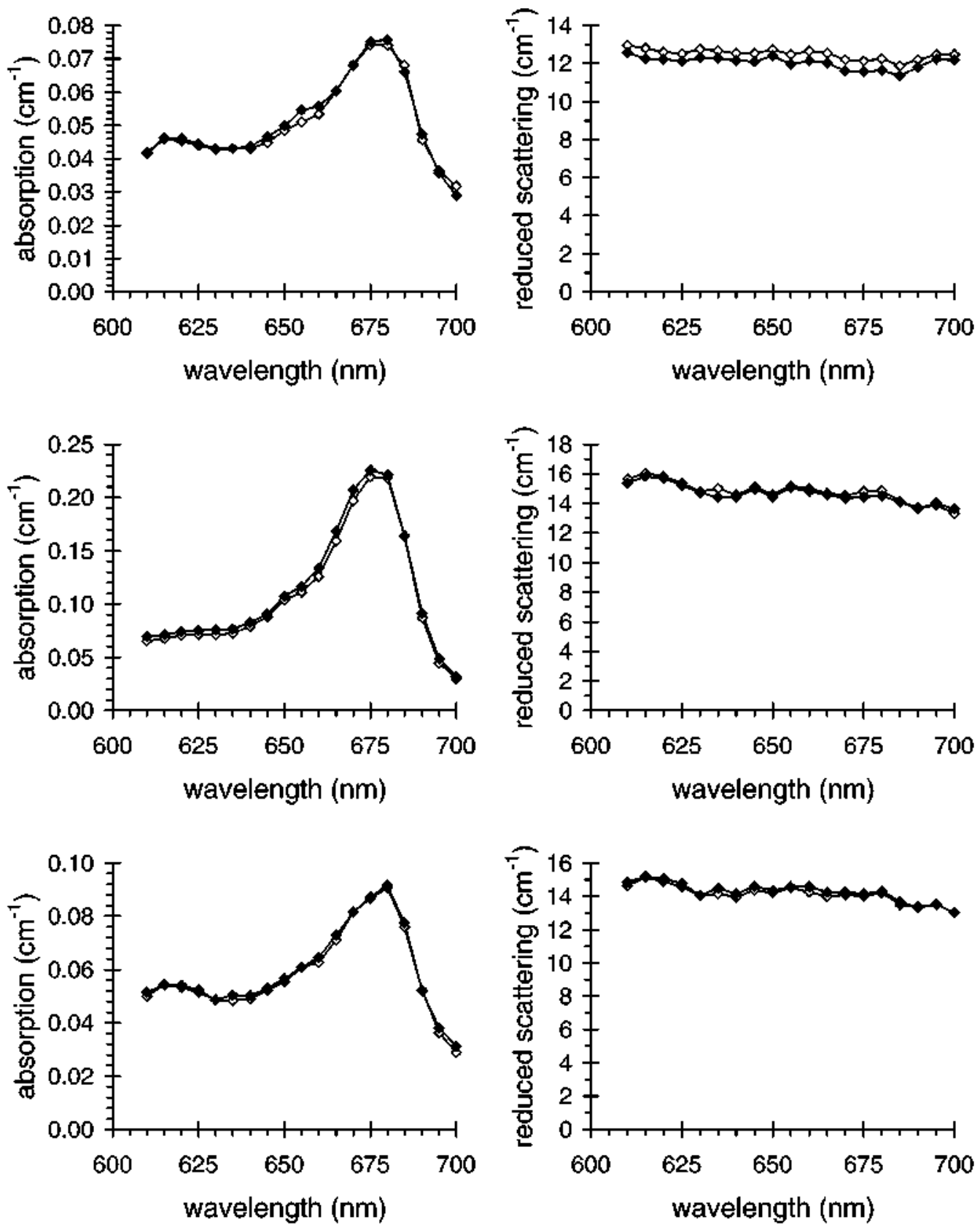

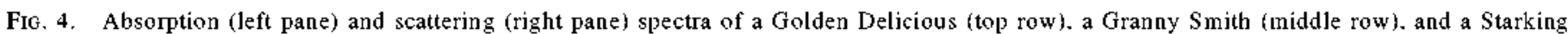
Delicious apple (bottom row). Before (filled diamonds), and after (empty diamonds) skin removal.
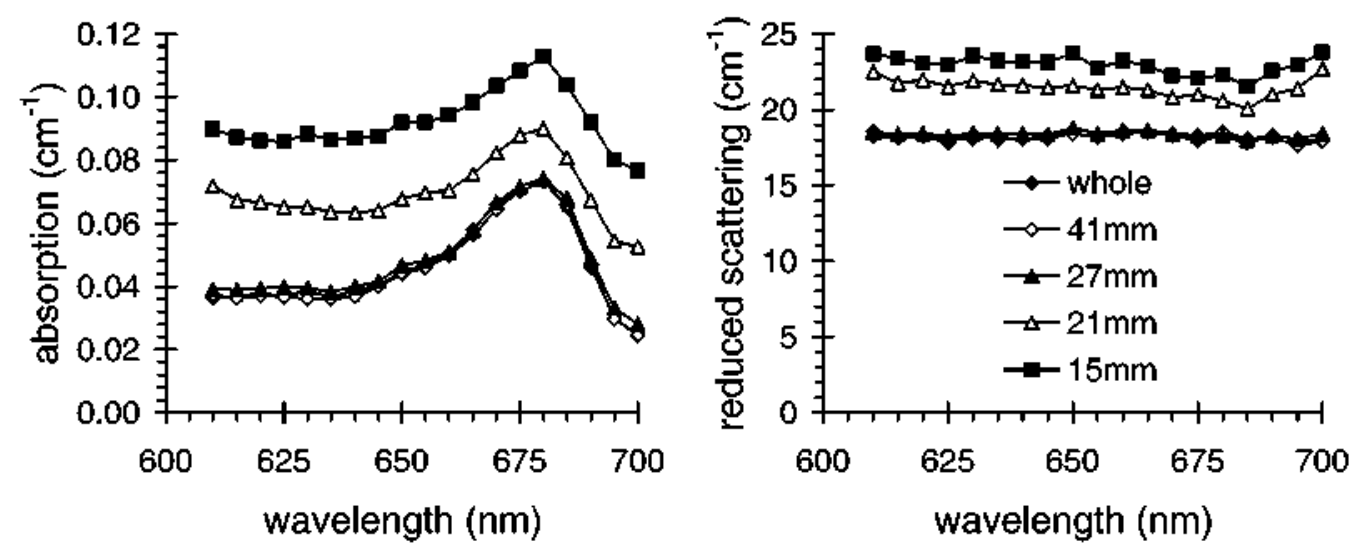

Fig. 5. Absorption (left pane) and scattering (right pane) spectra for different thicknesses of tissue from a Starking Delicious apple. 


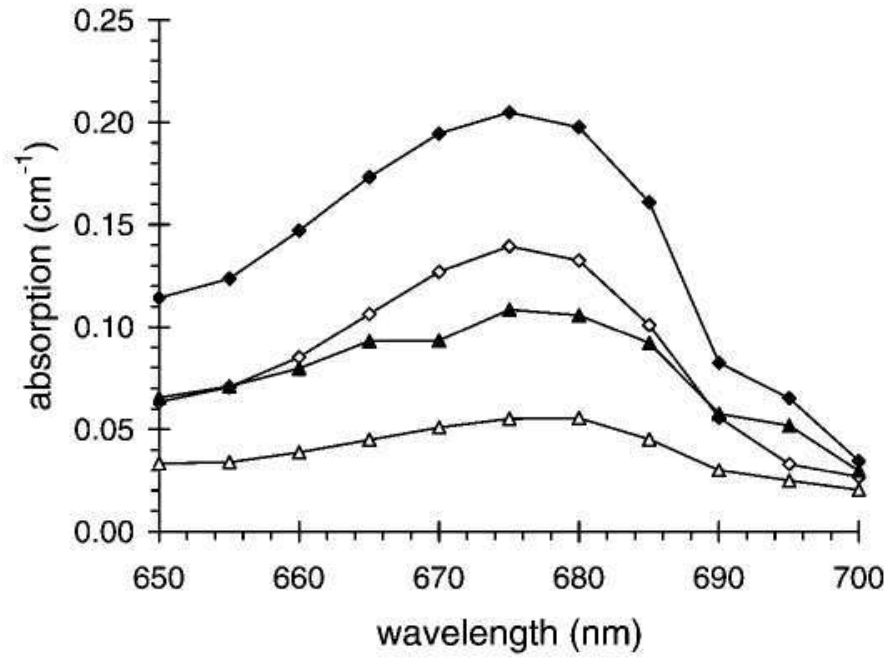

FIG. 6. Changes in the absorption spectrum due to ripening in Golden Delicious. Apples with green background color (diamonds) and yellow background color (triangles), measured at time 0 (filled symbols) and after 4 days of storage at room temperature (empty symbols).

for the more absorbing apple and of $50 \%$ for the less green one.

To prove the applicability of the technique in real-life applications, we sent the compact prototype for TRS measurements to Horticulture Research International, where it was tested on a picking date experiment, to check the tracking of maturity stage in apples. Fruits from the Gala variety were harvested from the same orchard at three different picking dates (pick $1=15$ September, pick $2=25$ September, and pick $3=9$ October), stored under controlled atmosphere at $1.5{ }^{\circ} \mathrm{C}$ for 7 months, and then measured all together with the use of the prototype. For each fruit, four equally spaced positions on the equatorial plane were measured and averaged. Results are presented in Fig. 7, where every fruit is coded by its $\mu_{\mathrm{a}}$ and $\mu_{\mathrm{s}}^{\prime}$ at $672 \mathrm{~nm}$. The measured $\mu_{\mathrm{a}}$ decreases, passing from pick 1 (black diamonds) to pick 2 (gray diamonds) and to pick 3 (white diamonds), indicating a decrease in CHL content. Also the scattering coefficient is somehow related to the picking date with a general decrease for latest harvest.

\section{DISCUSSION}

In a previous paper we demonstrated the applicability of TRS for the nondestructive and noninvasive measurement of the optical properties in fruits. ${ }^{13}$ In this work we have studied the key features of this novel approach that could eventually determine its possible use as a valid diagnostic tool on agriculture produce.

A first attribute of the technique is the independence of the measurement from the color of the skin. A thin absorbing layer behaves like an attenuating filter, without affecting the shape of the TRS curve. At a given point on the fruit surface, the intensity of re-emitted light is decreased due to the skin absorption, but the shape of the time-dispersion curve is minimally affected because photons spend little time traveling through the skin. This property is of great impact since most of the available nondestructive tests for quality control rely on the exter-

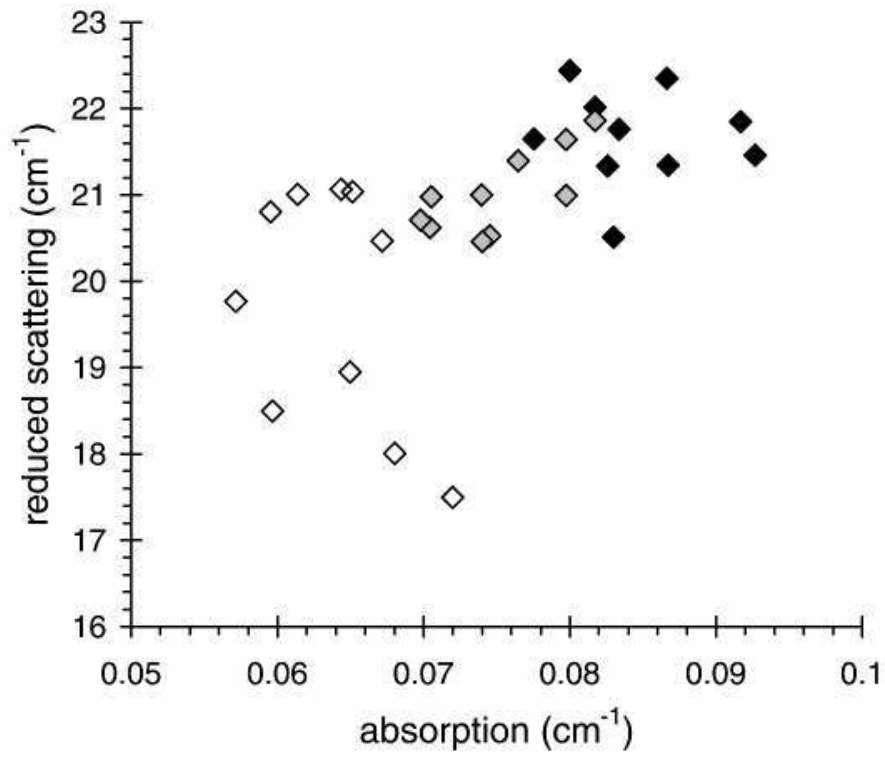

Fig. 7. Plot of the absorption and scattering measurements of 30 apples taken from a Gala cultivar at subsequent harvest dates: pick 1 (black diamonds), pick 2 (gray diamonds), pick 3 (white diamonds), and measured all together with the prototype after 7 months storage under controlled atmosphere.

nal appearance of the fruit, while taste is more related to the pulp.

A further aspect to be considered is the depth reached by TRS. Photons involved in a TRS measurement probe a region of the pulp of the fruit with a typical banana shape. ${ }^{19}$ Thus, the measured coefficients roughly correspond to the average of the optical properties in this region. In particular, for the tested Granny Smith applei.e., for $\mu_{\mathrm{s}}^{\prime} \approx 18 \mathrm{~cm}^{-1}$, and $\mu_{\mathrm{a}} \approx 0.07 \mathrm{~cm}^{-1}$ at $675 \mathrm{~nm}-$ we have shown that photons travel in the fruit deeper than $2 \mathrm{~cm}$ beneath the skin. Although this is not a direct estimation of the penetration depth of the technique, it proves that the TRS measurement is not confined to the surface of the fruit. Moreover, as the penetration depth depends on the optical properties of the fruits, deeper penetration can be expected where absorption and/or scattering are lower. For lower values of $\mu_{\mathrm{a}}$ (e.g., on the tails of the CHL absorption peak) the probed region would be deeper. Actually, in Fig. 5 the largest effects are observed on the tails of the spectrum.

We have shown that marked changes in CHL absorption are associated with the ripening process at room temperature. Under the experimental conditions proposed in this study, the TRS technique is capable of tracking a relative change of $\mu_{\mathrm{a}}$ to a precision of $2 \% .{ }^{18}$ Thus, a high sensitivity in monitoring internal processes of fruit that involve variations in the absorption coefficient can be expected.

We have used the TRS technique to measure the absorption and scattering properties of different apple varieties in the spectral region around the CHL absorption peak. The technique can also be applied to other turbid fruits or vegetables (for example, we have measured pears, peaches, nectarines, melons, kiwifruits, potatoes, and tomatoes), or to other diffusive biological media (e.g., bulbs). On the other hand, the interpretation of TRS data presents more difficulties whenever the scattering in 
the pulp is too low (e.g., oranges) or the fruit too small (e.g.. strawberries).

The use of the optical properties of the pulp of fruits and vegetables for the assessment of fruit internal quality has still to be investigated. More studies are required to correlate the neasured optical properties to other chemicals or physical parameters of the fruit such as soluble solids (sugar), acidity, or firmness. Since TRS permits the measurement of the absorption spectrum of the pulp independent of the scattering properties, it may be possible to detect other absorbing substances such as anthocyanins in the visible region or sugar and water in the near-infrared region. This technique might be suitable for following the ripening process pre-harvest or for monitoring fruit changes during long-term storage. Scattering inside a fruit is mainly due to refractive index mismatches between liquids and membranes. Thus, the mean scattering coefficient could provide information on the internal structure, as suggested by a recent study on kiwifruits. ${ }^{20}$ In our work, changes in the scattering coefficient were related to the stage of maturity and to the ripening process and could contribute to monitor them.

A possible criticism of the usefulness of TRS for applications in agriculture is the cost and complexity of the instrumentation, especially whenever more than one wavelength is needed. However, rapid progress in optoelectronics, particularly in telecommunications, has led to considerable growth in instrumentation for time-resolved measurements so that the development of a compact and low-cost time-resolved instrument is now feasible. A first prototype, working with semiconductor lasers, a compact photomultiplier, and all-fiber optics. that can be used as a stand alone, portable instrument, was built in our laboratory. The instrument was shipped to the UK and tested on stored apples. As presented in the previous section. there was good correspondence between the harvest date and the measured $\mu_{\mathrm{s}}$ and $\mu_{s}^{\prime}$, suggesting that the compact prototype, although less versatile and complete then the laboratory setup, is capable of tracking maturity staging in post-harvest produce. Acquisition time was set to $4 \mathrm{~s}$ for the data presented, yet an increase in the diode power, as well as in the collection efficiency by means of optical bundles, could reduce the measurement time to hundreds of milliseconds to meet high-speed processing of automated fruit-sorting lines.

Clearly, many technical aspects still need to be solved before an industrial application can take place. Most of all, the fruit characterization in terms of pulp optical properties has to be compared to the presently accepted estimators of fruit quality, which are sugar content, acidity, and firmness.

\section{CONCLUSION}

In conclusion, we have demonstrated the key features of TRS applied on apples for the noninvasive detection of the absorption and reduced scattering coefficients. With an interfiber distance of $1.5 \mathrm{~cm}$, the measurement is sensitive to the optical properties of the pulp down to a depth of approximately $2 \mathrm{~cm}$ and is not influenced by the skin. In the wavelength range from 610 to $700 \mathrm{~nm}$. the technique is able to reveal the absorption spectrum of CHL and is sensible to changes in the optical properties due to ripening and storage. Finally, we have developed a compact and low-cost prototype working at a selected wavelength that has demonstrated interesting potentialities for fruit sorting or grading.

\section{ACKNOWLEDGMENT}

This work was partially supported by the EC Grant FAIR CT96-1060.

1. A broad review of different nondestructive or minimally invasive techniques for fruit quality estimation can be found in $P$. Chen. "Quality Evaluation Technology for Agricultural Products", Proc. International Conference on Agricultural Machinery Engineering, Seoul, Korea (1996), pp. 171-204.

2. M. Bergevin, C. N. N'Soukpoékossi, R. M. Leblanc, and C. Willemot. Appl. Spectrosc. 49, 397 (1995).

3. M. Benady, J. E. Simon, D. J. Charles. and G. E. Miles, Trans. ASAE 38, 251 (1995).

4. A. Mizrach. N, Galili, and G. Rosenhouse, Trans. ASAE 32, 2053 (1989).

5. P. Chen. M. J, McCarthy, and R. Kauten. Trans. ASAE 32, 1747 (1989).

6. M. J, Delwiche, S, Tang, and J. W. Rumsey. Trans. ASAE 30, 1873 (1987).

7. S. Gunasekaran. M. R. Paulsen, and G. C. Shove. J, Agric. Eng. Res. 32, 209 (1985).

8. For a comprehensive treatment of light scattering. see A. Ishimaru, Wave Propagation and Scattering in Random Media, Vol.1, Single Scattering and Transport Theory (Academic Press, New York, 1978).

9. S. L. Jacques, IEEE Trans. Biomed. Eng. 36, 1155 (1989).

10. M. S. Patterson, B. Chance, and B. C. Wilson. Appl. Opt. 28, 2331 (1989).

11. S. J. Madsen, B. C. Wilson, M. S. Patterson, Y. D. Park, S. L. Jacques, and Y. Hefetz, Appl. Opt. 31, 3509 (1992).

12. A. Yodh and B. Chance, Phys. Today 48, 34 (1995), and references therein.

13. R. Cubeddu, C. D'Andrea, A. Pifferi, P. Taroni, A. Torricelli, G. Valentini, C. Dover, D. Johnson, M. Ruiz-Altisent, and C. Valero. Appl. Opt. 40,538 (2001).

14. R. C. Haskell, L. O, Svasaand, T.-T. Tsay, T.-C, Feng, M. S, McAdams, and B. J. Tromberg. J, Opt. Soc. Am. A 11, 2727 (1994).

15. K. Furutsu and Y. Yamada, Phys. Rev, E 50, 3634 (1994).

16. W. H. Press, S. A. Teukolsky. W. T. Vetterling. and B. P. Flannery, Nomerical Recipes in C: The Art of Scientific Computing (Cambridge University Press, New York, 1992).

17. R. Cubeddu, M. Musolino, A. Pifferi, P. Taroni. and G, Valentini. IEEE J. Quantum Electron, 30, 2421 (1994).

18. R. Cubeddu, A. Pifferi. P. Taroni. A. Torricelli. and G. Valentini. Med. Phys. 23, 1625 (1996).

19. S. Feng, F. Zeng, and B. Chance, Appl. Opt. 34, 3826 (1995).

20. V. A. McGlone, H. Abe, and S. Kawano. J. Near Infrared Spectrosc. 5. $83(1997)$. 